\title{
Importancia de la enseñanza de la memoria histórica en el contexto colombiano
}

\section{Importance of the teaching of historical memory in the colombian context}

\author{
Nercy Zuleima Moreno Báez² \\ Universidad Pedagógica y Tecnológica de \\ Colombia
}

\section{RESUMEN}

El presente artículo reporta un análisis de la importancia de la enseñanza de la Memoria Histórica en el contexto colombiano, partiendo de la relevancia de la memoria; asimismo se realiza una revisión teórica de algunos referentes de Memoria Histórica, que han contribuido a la construcción de nuevas reflexiones en las distintas sociedades; de la misma manera se plantea la necesidad de la participación de toda la sociedad en el proceso de construcción de memoria, para que la voz de las víctimas no quede invisibilizada y así las consecuencias

1 Artículo de Reflexión. Hace parte del trabajo de grado de la Maestría en Derechos Humanos, titulada Análisis de la Contribución de los Monumentos Conmemorativos como forma de Reparación Simbólica a las Víctimas del Conflicto Armado y su Importancia en la Construcción de Memoria Histórica en Colombia.

$2 \quad$ Magíster (C) en Derechos Humanos, Universidad Pedagógica y Tecnológica de Colombia UPTC. Especialista en Pedagogía de los Derechos Humanos, UPTC. Licenciada en Ciencias Sociales, UPTC. Docente Secretaria de Educación de Boyacá. Orcid ID: https://orcid.org/0000-00020072-7918 Correo electrónico: nercymb20@gmail.com. de la violencia en los más de cincuenta años de conflicto por parte de diferentes actores incluyendo al Estado, que ha dejado huellas en varias generaciones, no quede en el olvido. De igual modo se hace mención a lo concerniente a la importancia de la enseñanza de la Memoria Histórica desde los lugares de memoria, como los museos memoriales y monumentos conmemorativos, en donde la pedagogía de la memoria es un instrumento fundamental en el proceso de enseñanza-aprendizaje; así como también el papel fundamental que cumplen los maestros en el proceso de crear nuevas estrategias pedagógicas y didácticas que permitan un aprendizaje significativo.

PALABRAS CLAVE: Memoria Histórica, Enseñanza, Aprendizaje Significativo, Lugares de Memoria, Víctimas. 


\section{ABSTRACT}

This article reports an analysis of the importance of the teaching of Historical Memory in the Colombian context, starting from the relevance of memory as one of the main functions of the brain, in charge of reviving all kinds of memories; Likewise, a theoretical review of some references of Historical Memory is carried out, which have contributed to the construction of new reflections in the different societies. In the same way, the need for the participation of the entire society in the process of memory construction is raised, so that the violent events that occurred in more than fifty years of conflict by different actors, including the State, are not forgotten. that has left traces in several generations. Similarly, mention is made of the importance of the teaching of Historical Memory from places of memory, such as memorial museums and commemorative monuments, where memory pedagogies are a fundamental instrument in the teaching process. learning; as well as the fundamental role that teachers play in the process of creating new pedagogical and didactic strategies that allow meaningful learning.

KEYWORDS: Historical Memory, Teaching, Meaningful Learning, Places of Memory, Victims.

\section{INTRODUCCIÓN}

Analizar la importancia de la enseñanza de Memoria Histórica en el contexto colombiano se hace cada vez más necesaria, ya que Colombia ha sido un país que ha vivido y continúa viviendo las consecuencias de más de cincuenta años de conflicto entre distintos actores, y aún continúa dejando grandes huellas en las mentes, cuerpos de las víctimas, sus comunidades y territorios; asimismo, ha afectado profundamente el ámbito académico por los asesinatos, las desapariciones o el desplazamiento forzado de líderes estudiantiles y de grandes pensadores de la realidad colombiana; lo que hace que se genere la necesidad de cultivar la memoria histórica. Así las cosas, el problema que se evidencia es el desconocimiento generalizado de la historia del conflicto armado y la indiferencia de la sociedad frente a las secuelas que han dejado los hechos violentos provocados por el conflicto en nuestro país, ya que ante los diferentes cambios sociales y ante la era tecnológica en la que nos encontramos, en donde cada día la información cambia, de manera que dejan en el olvido hechos importantes.

De acuerdo con lo expuesto, se plantea, ¿de qué manera es posible desarrollar una cultura de memoria histórica que perdure, dignifique a la víctima y proporcione el no olvido y la no repetición? Para resolver el interrogante formulado, se señala como objetivo de este artículo determinar la manera más eficaz de implementar una cultura de la memoria histórica en nuestro País.

Bajo el entendido de que la única respuesta posible a la pregunta es la educación, se analizará la labor de las escuelas frente a la enseñanza de la memoria histórica, pues es imprescindible su rol, de formar ciudadanos críticos, cuidadores y conservadores de la paz; asimismo se plantea la importancia de los lugares de memoria, como museos memoriales y monumentos conmemorativos ya que también realizan un importante aporte a la construcción de memoria y a la reparación simbólica de las víctimas.

La metodología utilizada fue de tipo descriptivo con un enfoque cualitativo donde se realizó un análisis histórico, a través del estudio del conflicto armado y la forma como se enseña en las aulas en Colombia la memoria histórica.

\section{DESARROLLO}

En primera medida, se debe aclarar ¿qué es Memoria Histórica? y para ello se toma el concepto del Centro Nacional de Memoria Histórica (CNMH) (s.f.) la define como "un 
vehículo para el esclarecimiento de los hechos violentos, la dignificación de las voces de las víctimas y la construcción de una paz sostenible en los territorios", de igual modo el $\mathrm{CNMH}$ plantea que tiene como objetivo "la creación de una narrativa integradora e incluyente que ofrezca las razones del surgimiento y evolución el conflicto armado interno, evidenciando las memorias y resistencias de las víctimas y sus comunidades, al igual que los relatos que han sido silenciados". $Y$ es así como se puede contribuir a lograr espacios de diálogo donde intervengan no solo el Estado, sino también la sociedad y las futuras generaciones que merecen un escenario distinto al de la guerra.

Además, es importante establecer el concepto de memoria, donde es definida por el Foro Revista de Derecho (2020), como una "palabra polisémica que abarca un abanico de sentidos, significantes y significados que ha sido abordada por varias disciplinas como la neurobiología, antropología, psicología, sociología y las ciencias políticas" (p.8), y es así como se evidencia su gran complejidad y su vital importancia en su esencial tarea de revivir todo tipo de recuerdos.

De igual modo Carrillo (2010), infiere que:

La memoria es sin duda un proceso cerebral notable, pues mediante él, el sistema nervioso codifica, organiza y almacena los sucesos pasados de tal forma que en ocasiones permite recordar de manera consiente, eventos en el pasado distante, tan vívidamente como si se experimentaran nuevamente (p. 86).

De acuerdo con lo anterior, la memoria permite recordar sucesos del pasado, ya sean eventos que provoquen manifestaciones de alegría, o por el contrario hechos que han generado algún tipo de dolor. Otro de los referentes conceptuales importantes en cuanto a memoria es Halbwachs (1968), quien menciona que, en cuanto a la construcción académico-política, la memoria como noción tiene unos fundamentos europeos, los cuales vienen a darse a mediados del siglo $\mathrm{XX}$, que corresponde al espacio de las guerras mundiales, es por esto que uno de los elementos importantes para abordar es la noción del autor anteriormente nombrado, quien plantea que no hay en la memoria un vacío dominante, partiendo de que toda imagen se relaciona con un recuerdo ya sea puro y simple, donde existe un carácter histórico fuera del individuo.

En este mismo sentido, es importante establecer la relación de la memoria con la historia oral, pues, así como lo manifiesta Rueda (2013), fue un elemento esencial en las antiguas sociedades como la griega, en donde la correlación social y el pasado cultural, instauraron una forma de heredar información a diferentes generaciones, como lo fueron las distintas guerras en donde sus acontecimientos fueron evidentes debido al relato oral y es donde las versiones de los testigos cobran relevancia, ya que en este periodo tener acceso a fuentes escritas resultaba difícil para los historiadores de la época.

Ya durante el medioevo, los historiadores resaltan la importancia que tuvo la memoria, pues se convirtió en un aspecto importante para la reproducción de documentos como lo fueron las diversas obras literarias y las múltiples crónicas, reconstruidas también por medio de relatos orales, teniendo en cuenta que sentían la necesidad de escribir lo que vivían, y es así como se construye la historia por medio de memorias individuales y colectivas. De igual forma, se hace mención a las fuentes históricas orales en donde son tomadas como fuentes narrativas y se plantea que lo verdaderamente significativo de la memoria no es que sea un depósito de hechos, sino que es un dinámico proceso de creación de significados, dándole sentido al pasado y una forma a sus vidas según lo argumenta Portelli (1991). 


\section{REFERENTES SOBRE MEMORIA HISTÓRICA}

En este sentido, pasamos a mencionar a uno de los referentes de Memoria Histórica más sobresalientes, como lo es el historiador francés Pierre Nora (1984), quien establece ciertas disparidades entre memoria e historia, mencionando que la historia es la vida constantemente encaminada entre diversos colectivos y que se encuentran en constante cambio, que esta accesible a la lógica del recuerdo y de la amnesia inconsciente y que es vulnerable a ser manipulada, que se sostiene de recuerdos globales, particulares o simbólicos, mientras que la historia es el restablecimiento de lo que ya no es, en una representación del pasado.

De igual forma, Nora (1984), plantea que los sitios de memoria nacen y viven del sentir de que no hay memoria espontánea, de tal modo que se deben organizar archivos, conservar las conmemoraciones, emprender diferentes actos como realizar actas, celebraciones, ya que estos procedimientos no son naturales y por lo tanto necesitan estar en contacto activo con la sociedad.

Con lo anterior se infiere, la necesidad de que la sociedad debe participar en el proceso de construcción de memoria, con diferentes actos donde se pueda rememorar constantemente y no solo quedarse como un sujeto aislado.

Del mismo modo, es importante señalar la nueva labor de la sociedad frente a la memoria, como lo propone Almanza (2013), quien menciona que la indiferencia permite evitar el compromiso con los hechos ocurridos y así establecer o favorecer el olvido y el progreso, y de esta manera se transforma el discurso permitiendo ocultar o minimizar las víctimas para que así no inquieten ni alteren la tranquilidad, pues al señalar a los afectados como distintos y no permitir que sean aceptados por una sociedad ha generado que se transforme, negar los acontecimientos ocurridos en dicha sociedad permite que ésta tenga una regresión en el reconocimiento de sus derechos humanos.

Con base en lo anterior, conviene mencionar las acepciones para Memoria Histórica, Memoria Colectiva y Memoria Individual en donde Halbwachs, citado por Betancourt (2004), sitúa los hechos personales de la memoria, tanto los eventos de tipo individual como los eventos que se originan de la interacción con otros grupos en donde convivimos, así como también las relaciones que se establecen entre estos grupos, así el autor define:

> Memoria histórica: supone la recolección de los datos proporcionados por el presente de la vida social y proyectada sobre el pasado reinventado.

> Memoria Colectiva: es la que recompone mágicamente el pasado y cuyos recuerdos se remiten a la experiencia de una comunidad o que un grupo puede llegar a legar a un grupo o grupos de individuos.

> Memoria Individual: en tanto que esta se opone (enfrenta) a la memoria colectiva, es una condición necesaria y suficiente para llamar al reconocimiento de los recuerdos; nuestra memoria se ayuda de otras, pero no es suficiente con que ella nos aporte testimonio (p.126).

Es así como la interacción entre lo histórico, lo colectivo y lo individual se crea a partir de la experiencia de estos grupos, donde cada uno se construye de acuerdo a sus vivencias, siempre estando condicionadas a fenómenos sociales, colectivos y públicos.

Según Betancourt (2004), se establece el pensamiento de que la Memoria Histórica, la 
Memoria Colectiva y la Memoria Individual se generan a partir de dos momentos significativos, la experiencia percibida y la experiencia vivida, en donde la experiencia vivida abarca esos saberes históricos, sociales y culturales que las personas o grupos sociales aprehenden a vivir en su vida, mientras que la experiencia percibida reúne los elementos históricos, sociales y culturales de los individuos o grupos, en donde adoptan del discurso político, religioso, de las ideas filosóficas o las diferentes opiniones culturales, un conocimiento formalizado, producido y acumulado.

Por otra parte, el Centro Nacional de Memoria Histórica (2018), alude el papel de la memoria histórica como un ejercicio político que involucra la clasificación de hechos y construcción de relatos sobre el pasado, que contiene consensos y disensos sobre lo que debe ser contado y considerado como la historia de una persona o un grupo, es por esto que la memoria es considerada como la forma en que se busca darle sentido a los hechos y concederle viabilidad para que sean relatados; pues en algunas ocasiones se logra vencer la forma como se recuerda, mientras que otros resisten el dominio desmedido del relato hegemónico, pues a mayor poderío político, mayor cabida de intervenir el contenido de la memoria. Por lo cual, se establece que no debe existir una intervención política en la construcción de memoria, ya que puede generar una manipulación de los hechos y por lo tanto provocar revictimización de los afectados.

Otro aspecto importante, es el aporte de los lugares de memoria a la construcción de memoria histórica, como lo son los museos, sitios de memoria y los monumentos conmemorativos, los cuales se han creado a partir de la necesidad de las víctimas de que se sepa lo ocurrido con sus familias, comunidades y territorios, en donde no solo han sido implementados en Colombia sino también en distintos lugares del mundo que de igual manera han sido afectados por múltiples razones.

En el caso de los monumentos conmemorativos en Colombia, han sido un instrumento importante en la tarea de la reparación simbólica, entendiéndose ésta según lo estipulado en el marco de la Ley 1448 de 2011 como "toda prestación realizada en favor de las víctimas o de la comunidad en general, que tienda a asegurar la preservación de la memoria histórica, la no repetición de los hechos victimizantes y el restablecimiento de la dignidad de las víctimas" (Art 141).

En consecuencia, los lugares de memoria cumplen una función vital en la construcción de memoria histórica, ya que como lo menciona Mora (2013), en éstos lugares se realizan las reflexiones de ciertas comunidades de acuerdo a sus experiencias y a su simbolismo; pues en distintos territorios debido a las políticas e ideologías se establece, qué se recuerda, qué se olvida y en función de qué recordamos. Lo que crea la necesidad de que toda la sociedad tome enserio a estos lugares ya que han sido creados para la enseñanza de la historia violenta de nuestro país y así no se permita su olvido.

\section{REFERENTES EN LA ENSEÑANZA DE LA MEMORIA HISTÓRICA EN COLOMBIA}

Para un país que intenta por distintos medios superar un pasado de conflicto y violencia es importante abordar la memoria histórica desde el ámbito educativo, ya que es uno de los lugares en donde se crean las distintas reflexiones en torno a los hechos del pasado, con las actuales y futuras generaciones, que tendrán como labor fundamental continuar construyendo la paz; ya que es evidente el desconocimiento e indiferencia por parte no solo de algunos jóvenes sino también de comunidades que al no haber vivenciado directamente las consecuencias del conflicto armado, permiten el olvido y facilitan su repetición. 
Por esta razón, se aborda el concepto de aprendizaje significativo, en donde Moreira citado por Londoño \& Carvajal (2015), refiere que es "un aprendizaje auténtico que se genera en la medida en que integra nuevos conocimientos a la estructura cognitiva y experiencial del sujeto que aprende y que ha desarrollado a través de su historia de vida" (p.7). Por lo cual, para que exista un verdadero aprendizaje significativo es necesario que los estudiantes tengan conocimiento acerca de su historia y los fenómenos sociales de su entorno, para así lograr construir un pensamiento crítico y reflexivo frente a las distintas situaciones del país. Por lo que, desde la Pedagogía Crítica, Abraham Magendzo (2003), manifiesta que "el objetivo es desarrollar una conciencia crítica en el estudiante y promover la acción social para superar estructuras sociales opresivas" (p. 3). Por lo tanto, para poder desarrollar esta conciencia crítica, se le deben brindar a los educandos las herramientas necesarias, para la construcción del conocimiento; que sean atractivas e interesantes, y que contribuyan a su formación integral.

Conviene definir el concepto de la pedagogía de la memoria, en donde Capra (s.f.) señala:

la articulación de esas prácticas referidas a los procesos de significación y apropiación de nuestra historia reciente, por parte de sujetos en situación de recorrido por el espacio de la memoria. Se articula con nociones referidas al rol activo por parte de los sujetos en el proceso de construcción del saber; con una perspectiva dialogal y circular del uso de la palabra; con definiciones entorno a la imposibilidad de la neutralidad en el posicionamiento frente a la realidad observada, conversada y resignificada (p.2).

De manera que no deben ser sujetos indiferentes a los distintos fenómenos sociales que se encuentran en su contexto, por el contrario, deben ser participativos y convertirse en agentes de cambio.

Por ende, es relevante mencionar el objetivo de la pedagogía de la memoria, partiendo del planteamiento de Ortega \& Castro (2015), en donde describen su importancia, mencionando que "permite la posibilidad de aprendernos, reconocernos yenseñarnos distintos, al identificar y analizar otras formas de experiencias, deseos, esperanzas y resistencias" (p. 29).

Es indispensable mencionar uno los referentes más importantes en el trabajo de pedagogía de la memoria en Colombia, como lo es el Centro Nacional de Memoria Histórica (CNMH), que junto con otros lugares de memoria, desempeña una labor clave en distintos lugares del territorio nacional, tanto en los que vivieron la violencia como en los territorios que no estuvieron directamente implicados en el conflicto armado, pues es en estos últimos donde más se evidencia la discriminación e indiferencia hacia las víctimas; por eso por medio de actividades itinerantes el $\mathrm{CNMH}$ da a conocer qué paso en nuestro país, en estos más de 50 años de conflicto, asimismo la Red de lugares de memoria de Colombia, los cuales cumplen una función fundamental, no solo porque algunos vivieron el conflicto, sino también porque permiten que se conozca la verdad de lo sucedido, permite que se visibilice la voz de las víctimas por medio de sus testimonios, en donde se realiza un retrato de todas las caras de la violencia, por medio de talleres vivenciales en donde quienes los dirigen son las mismas mujeres que fueron víctimas de violencia sexual, los mismos campesinos que fueron desplazados y de jóvenes que fueron obligados a presenciar como torturaban 0 asesinaban a sus padres, hermanos o vecinos, y que fueron obligados a dejar sus tierras y a iniciar de nuevo en lugares completamente extraños para ellos, volviendo a ser víctimas de discriminación en las ciudades. 


\section{IMPORTANCIA DE LA ENSEÑANZA DE MEMORIA HISTÓRICA}

Existen múltiples razones y muchas ventajas pedagógicas para abordar la memoria histórica con los estudiantes en Colombia, ya que permite desarrollar empatía, pensamiento crítico, evaluar distintas perspectivas de la historia, así como también una de las razones de trabajar memoria histórica y que consideramos una de las más importantes como lo es desnaturalizar la guerra, ya que muchos niños y jóvenes no tiene conocimiento acerca de la historia del conflicto, o simplemente lo consideran como algo normal, debido a que son generaciones que nacieron en un país en guerra, lo cual hace que la normalicen.

Por lo tanto, se debe realizar una verdadera sensibilización y conocimiento de los hechos de violencia que marcaron y siguen marcando la historia de nuestro país, en donde el proceso de enseñanza-aprendizaje sea significativo, de manera que se formen ciudadanos críticos, cuidadores y conservadores de la paz, en donde esto se lleve a una verdadera cultura de paz, en la que las actuales y futuras generaciones, dejen de normalizar los hechos violentos en los que se ha estado inmerso durante años y se lleve a construir nuevas prácticas de paz y nuevas formas de relacionarnos.

Para lograr este propósito, es necesario que los docentes formulen nuevas estrategias pedagógicas y didácticas, en donde realicen una aproximación de los educandos a la historia y al contexto actual colombiano, no solo desde el aula de clase, pues para estudiar los fenómenos sociales es necesario que luego de conocer teóricamente, y analizar los hechos y consecuencias del conflicto, se tengan experiencias formativas, como las prácticas pedagógicas a lugares de memoria, como los museos memoriales en donde de una manera didáctica muestran la realidad y fomentan la construcción de memoria histórica, para que como agentes de cambio en un futuro cercano, favorezcan la construcción de paz y la garantía de no repetición; de igual forma con este tipo de pedagogía se favorece en los niños y jóvenes el desarrollo de competencias por medio del estudio, análisis y comprensión del conflicto armado colombiano.

Así como lo señala Pagés, citado por Aponte (2017) "recurrir a la memoria individual, al recuerdo de hechos, acontecimientos, problemas del pasado ha sido una de las características principales de aquel profesorado, que ha intentado innovar sus prácticas docentes al menos desde la década de los años setenta del pasado siglo XX" (p.7). Argumentación que se demuestra en el quehacer docente, al percatar a los estudiantes de que ellos son importantes agentes de cambio y que en un futuro cercano pueden influir en las decisiones del país, pues al conocer su entorno y conocer acerca de los diferentes fenómenos históricos, políticos y culturales que ocurren a su alrededor pueden contribuir en la construcción de una mejor sociedad.

Adicionalmente, el docente Carlos Barrera ("en prensa"), señala "el destino de los pueblos se traza conociendo su pasado, en él está la información de sus tradiciones y su ADN social, recogido en las mentalidades colectivas, pretendiendo construir imaginarios de progreso" (p.12).

Por otro lado, dentro del marco normativo, es esencial mencionar de manera general, una de las leyes creadas durante el Acuerdo Final para la Terminación del Conflicto y la Construcción de una Paz Estable y Duradera firmado en el 2016, con el grupo guerrillero FARC-EP, la cual ha aportado a la reparación de las víctimas y en la construcción de memoria histórica en Colombia, como lo es la Ley 1448 de 2011, en donde en sus artículos 142 y 143 establece que: 
Artículo 142. Día Nacional de la Memoria y Solidaridad con las Víctimas. El 9 de abril de cada año, se realizará por parte del Estado colombiano, eventos de memoria y reconocimiento de los hechos que han victimizado a los colombianos y las colombianas. El congreso de la república se reunirá en pleno ese día para escuchar a las víctimas en una jornada de sesión permanente.

Artículo 143. Del Deber de Memoria del Estado. Se traduce en propiciar las garantías y condiciones necesarias para que la sociedad, a través de sus diferentes expresiones tales como víctimas, academia, centros de pensamiento, organizaciones sociales, organizaciones de víctimas y de derechos humanos, así como los organismos del Estado que cuenten con competencia, autonomía y recursos, puedan avanzar en ejercicios de reconstrucción de memoria como aporte a la realización del derecho a la verdad del que son titulares las víctimas y la sociedad en su conjunto (Ley 1448, 2011)

Es así, como de esta manera se le brinda a la Memoria Histórica, un espacio en donde se permite que la sociedad participe en la construcción de una paz estable, generando nuevas reflexiones, frente a qué no se debe permitir que estos hechos vuelvan a repetirse.

De modo que, el trabajo y responsabilidad frente a la construcción de memoria histórica no solo es del Estado, sino también de la sociedad, para que desde la escuela se genere y cultive un pensamiento crítico, que comprenda la historia y los hechos violentos de un país que solicita que se siga tejiendo la paz.

\section{CONCLUSIONES}

En un país en donde el conflicto armado ha dejado y continúa dejando grandes huellas en las mentes y cuerpos de las víctimas, sus comunidades y territorios, se requiere de manera fundamental la implementación de las pedagogías de la memoria en donde se formen individuos comprometidos con historia y memoria de su país y en donde se deje de normalizar la violencia. En este sentido, la única manera implementar una cultura de memoria histórica que perdure, dignifique a la víctima y proporcione el no olvido y la no repetición es a través del desarrollo de la pedagogía de la memoria.

Por lo tanto, cuando se habla de memoria, significa poner en primer nivel la voz de las víctimas, sus recuerdos y la manera como ellas vivieron los hechos en el marco del conflicto; para que las actuales y futuras generaciones comprendan cómo han aportado a la construcción de paz, desde el testimonio y desde su lucha por la reivindicación de sus derechos, enseñando como después de haber vivenciado las atrocidades de la guerra, han logrado la resiliencia y una construcción de memoria histórica.

Así como también, la importancia de las experiencias formativas y significativas, de niños, niñas y jóvenes, sobre el contexto en el que se ha desarrollado la violencia, por medio de la pedagogía en los lugares de memoria, como museos memoriales y monumentos conmemorativos, donde se facilita el proceso de enseñanza-aprendizaje, promueve competencias y genera reflexiones críticas y constructivas entorno a crecimiento como ciudadanos constructores de paz.

De manera que ante los cambios sociales y ante la era tecnológica en la que nos encontramos, nace la necesidad y obligación de cultivar la memoria, no solo desde la voz de las víctimas 
sino también de aquellas voces que nunca se escucharon, que se silenciaron; también la enseñanza de la memoria histórica desde los territorios, tanto los que vivenciaron el conflicto como en los territorios que no, para que se conozcan los hechos y daños causados, eliminando la indiferencia e indolencia, en donde también se incluye al Estado, pues el hecho de no haber vivenciado la violencia no significa que no haya existido.

\section{REFERENCIAS BIBLIOGRÁFICAS}

Almanza, T. (2013). La memoria de la experiencia como respuesta ética ante las víctimas. Revista de las Ciencias del Espiritu. Universidad de San Buenaventura: https://www.redalyc.org/articulo. oa?id=343529829002

Aponte, C. (2017). Memoria Histórica y Conflicto Armado: elementos para la construcción de una educación para la paz en la escuela colombiana. Revista Cambios y Permanencias: https://revistas.uis. edu.co/index.php/revistacyp/article/ view/7833/8005

Barrera, C. ("en prensa"). Memoria e Imaginarios Iconográficos sobre la Independencia, vistos a través de tres generaciones, en la larga duración (1910-2019).

Betancourt, D. (2004). Memoria individual, memoria colectiva y memoria histórica : lo secreto y lo escondido en la narración y el recuerdo. Bibiloteca Clacso: http:// biblioteca.clacso.edu.ar/Colombia/dcsupn/20121130052459/memoria.pdf

CNMH. (2018). Género y Memoria Histórica. CNMH: http://www. centrodememoriahistorica.gov.co/ micrositios/balances-jep/descargas/ balance-genero.pdf
Capra, M. (s.f). Los Espacios para la Memoria como espacios Educativos: Construyendo una Pedagogía de la Memoria:

https://apm.gov.ar/periplosdememorias/ materiales/23/AportesReflexion/ ConstrucPedagMemoria/ponencia $\% 20$ IDES $\% 20$-\%20Espacio $\% 20$ de $\% 20$ Memoria\%20La\%20Perla.pdf

Carrillo, P. (2010). Sistemas de memoria: reseña histórica,clasificación y conceptos actuales.Primera parte: Historia, taxonomía de la memoria, sistemas de memoria de largo plazo: la memoria semántica. Medigraphic: https://www. medigraphic.com/pdfs/salmen/sam2010/sam101j.pdf

Foro Revista de Derecho. (2020). Memoria, reparación simbólica y arte: la memoria como parte de la verdad. Foro Revista de Derecho: https://revistas.uasb.edu.ec/ index.php/foro/article/view/1285/1197

Halbwachs, M. (1968). Memoria Colectiva y Memoria Histórica. http://ih-vmcisreis.c.mad.interhost.com/REIS/PDF/ REIS_069_12.pdf

Nora, P. (1984). Entre Memoria e Historia: La problemática de los lugares. https:// www.comisionporlamemoria.org/ archivos/jovenesymemoria/bibliografia_ web/historia/Pierre.pdf

Ley 1448 . (10 de Junio de 2011). Ley de Víctimas del Conflicto Armado. Unidad de Víctimas : https://www. unidadvictimas.gov.co/sites/default/ files/documentosbiblioteca/ley-1448de-2011.pdf

Londoño, J. (2015). Pedagogías para la memoria histórica: reflexiones y consideraciones para un proceso de innovación en el aula. Ciudad Paz-Ando: 
https://revistas.udistrital.edu.co/index.php/cpaz/ article/download/8261/10594/

Magendzo. A. (2003). Pedagogía Critica y Educación en Derechos Humanos. Revista de Pedagogía Crítica:

https://www.researchgate.net/ publication/318844597_Pedagogia_ critica_y_educacion_en_derechos_ humanos

Mora, Y. (2013). Lugares de Memoria entre la tensión, la participación y la reflexión. Dialnet: https://dialnet.unirioja.es/ descarga/articulo/4780112.pdf

Ortega, P. \& Castrro, C. (2015). Pedagogía de la Memoria para un país amnésico. Universidad Pedagógica Nacional:http://editorial.pedagogica. edu.co/docs/files/Pedagogia $\% 20$ de $\% 20$ la $\% 20$ Memoria $\% 20$ - \% 20 sampler.pdf Rueda, J. (2013). Memoria histórica razonada".Una propuesta incluyente para las víctimas del conflicto armado interno colombiano. Historelo: Revista de Historia Regional y Local: https://www.researchgate.net/ publication/263511378_"Memoria_ historica_razonada"_Una_propuesta_ incluyente_para_las_victimas_del_ conflicto_armado_interno_colombiano/ fulltext/57aa9e6508ae3765c3b4f659/ Memoria-historica-razonada-Unapropuesta-incluyente-para

Portelli, A. (1991). Lo que hace diferente a la historia Oral. Comisión por la Memoria: https://www.comisionporlamemoria. org/archivos/archivo/archivooral/ bibliografia/PORTELLI\% 20 Alessandro, $\% 20$ Lo $\% 20$ que $\% 20$ hace $\% 20$ diferente $\% 20 a \% 201 a \% 20$ historia\%20oral.pdf 